\title{
An Industrial Vision System to Analyze the Wear of Cutting Tools
}

\author{
Christina Gillmann ${ }^{1}, \mathrm{a}$, Tobias Post ${ }^{1}, \mathrm{~b}$, \\ Benjamin Kirsch ${ }^{2}, \mathrm{c}$, Thomas Wischgoll ${ }^{3}$, , Jörg Hartig ${ }^{2}, \mathrm{e}$, \\ Bernd Hamann $^{4}$,f , Hans Hagen ${ }^{1}$, and Jan C. Aurich ${ }^{2}$,h \\ ${ }^{1}$ Computergraphics and $\mathrm{HCl}$, University of Kaiserslautern, Germany
}

${ }^{2}$ Institute for Manufacturing Technology and Production Systems, University of Kaiserslautern

${ }^{3}$ Advanced Visual Data Analysis, Wright State University, U.S.A.

${ }^{4}$ Department of Computer Science, University of California (UC Davis), U.S.A.

ac_gillma@cs.uni-kl.de, btpost@rhrk.uni-kl.de,

cbenjamin.kirsch@mv.uni-kl.de, ${ }^{\mathrm{d}}$ thomas.wischgoll@wright.edu, ejoerg.hartig@mv.uni-kl.de, fhamann@cs.ucdavis.edu, shagen@cs.uni-kl.de, hjan.aurich@mv.uni-kl.de

Keywords: Industrial Vision System, Image-based Wear Measurement, Comparative Visualization

\begin{abstract}
The wear behavior of cutting tools directly affects the quality of the machined part. The measurement and evaluation of wear is a time consuming process and is subjective. Therefore, an image-based wear measurement that can be computed automatically based on given image series of cutting tools and an objective way to review the resulting wear is presented in this paper. The presented method follows the industrial vision system pipeline where images of cutting tools are used as input which are then transformed through suitable image processing methods to prepare them for the computation of a novel image based wear measurement. For multiple cutting tool settings a comparative visualization of the wear measurement outputs is presented. The effectiveness of the presented approach is shown by applying the method to measure the wear of four different cutting tool shapes.
\end{abstract}

\section{Introduction}

Cutting tools, as applied in turning, milling or drilling processes, are exposed to wear due to the loads they are subjected to in cutting processes [1]. In the cutting zone, high temperatures and high forces emerge that result in abrasive and chemical wear of the tools. Commonly, the width of flank wear land at the flank face of the tool is used as the wear criteria [3]. The width of the flank wear land is determined manually via optical microscopes. This value is subjective and 1-Dimensional.

To solve this problem, an objective measure can be accomplished by different sensors [2] as shown in Section Related Work. These sensors can be expensive, require maintenance, and therefore are not available commonly in the shop floor. Instead, a technique is required that is independent from additional hardware and can be run in basic settings such as given by optical microscopes. The required measure should be flexible in terms of being able to handle different images of different cutting tools. In addition to that, the approach should have low computational costs so that it can be run on a common computer. Furthermore, this measure should be embedded in a visual system that allows the review of the computational output during operation. At last, this system has to provide an intuitive visualization of the evolved measure that allows an interactive exploration and comparison of wear behavior for decision making [4].

To achieve these goals, this paper presents a visual industrial system consisting of the four classical steps (image acquisition, image processing, feature extraction, and decision making), which is presented in Section Methods. As input, the system utilizes image series of cutting tools that can be monitored at different stages of the cutting process. This visual industrial system is designed to derive an objective measure based on the image input that is able to determine the wear of the captured cutting tools by applying approved and suitable image processing techniques for image registration 
and segmentation. The output of this algorithms can be used to define and calculate an image based measure for wear. This leads to an objective wear measure that can be observed over the cutting process without requiring additional sensors. To allow an intuitive and interactive handling of the wear measure, the algorithm output is encapsulated in a linked view visualization system that helps decision makers estimate the wear behavior of a cutting tools.

Therefore this paper contributes:

- An objective, image based metric of wear

- A visual industrial system for cutting tool observation

- A comparative visualization of cutting tool wear behavior

The industrial vision system presented in this study will be applied to the detection of the wear evolution of four cutting tools in Section Decision Making. The utilization of the developed vision system enables the statistical, geometrical and temporal analysis of the flank wear in an objective manner. The results will be discussed in Section Discussion and future directions are given in section Conclusion.

\section{Related Work}

This Section will give an overview over the state of the art of cutting tool wear analysis covering the available cutting measurement methodologies and visual industrial systems designed for cutting processes.

\section{Cutting Tool Wear Measurement}

Cutting tool wear can be described by various methods as outlined in [5]. The following Section will present the most common groups in relation to the presented method.

Various approaches measuring the wear of cutting tools based on additional sensors are available $[7,8,9,13]$. They can work on-line and are able to directly output a trustworthy and accurate measurement of wear. Their common drawback is the additional hardware required to obtain the wear measurement. These sensors can be expensive and require maintenance. In contrast to these methods, this paper presents a wear measurement that is computed solely on the basis of images captured from the cutting tools.

Statistical analysis $[11,12]$ is a further tool that can be used to obtain a wear measurement based on image inputs. Therefore, a statistical model is used to estimate the wear of cutting tools according to their material properties. Although these algorithms output promising results without requiring input images they are limited to cutting tools, with known physical properties. Therefore, the presented approach offers a wear measurement that can be computed independently from the cutting tool by reviewing images.

Stereoscopic images are another approach to estimating wear of cutting tools [17, 18]. This method utilizes images of different perspectives to reconstruct the cutting tool's changed shape. The difference of the original geometry to the changes in geometry is visualized by a colorcoding. Although this provides an accurate description of the wear surface, it requires a special image generation process which is not available in each setting. In contrast to that, the presented method is able to estimate the wear based on ordinary captured images.

\section{Visual Industrial Systems for Cutting Tools}

Visual industrial systems aim at monitoring a production process during its operating to provide on-line quality management [20]. The available visual industrial systems for cutting tools and their abilities are summarized below.

Systems working on-line during the operation of a production line without outputting a visual feedback of wear are widely used $[15,10]$. The goal of these systems is to estimate the cutting tool 
wear with the ultimate goal of determining when a cutting tool needs to be replaced by a new one. Although this system lowers the effort in process control, it is not able to provide a visual feedback to the decision maker. The result is a lack of trust in the monitoring system [4]. In contrast to the summarized approaches, this paper provides a direct visual feedback to the decision maker.

Visual industrial systems providing a visualization are also available $[14,6,16]$. These systems provide different outputs to identify the wear of a cutting tool and provide a visual alert for the decision maker when to exchange the cutting tool. Although this is a suitable first step to determining when intervention is required, these methods do not provide a relation between different cutting tool behaviors. As the selection of a proper cutting tool is a crucial factor to the cutting process's quality, this is a desired feature. Therefore, the presented visual industrial system can be extended to the ability of process monitoring of classical visual industrial systems to provide a comparative visualization of the cuttings tool's wear behavior.

\section{Methods}

The following Section presents a visual industrial system that is able to determine the flank wear of cutting tools at specific points of time based on images captured of the cutting tool and provides a comparison between different shapes of cutting tools. As shown in [20], an industrial vision system can be divided in four stages: image acquisition, image processing, feature extraction, and decision making. The following section will show how each of this steps can be performed using the presented method.

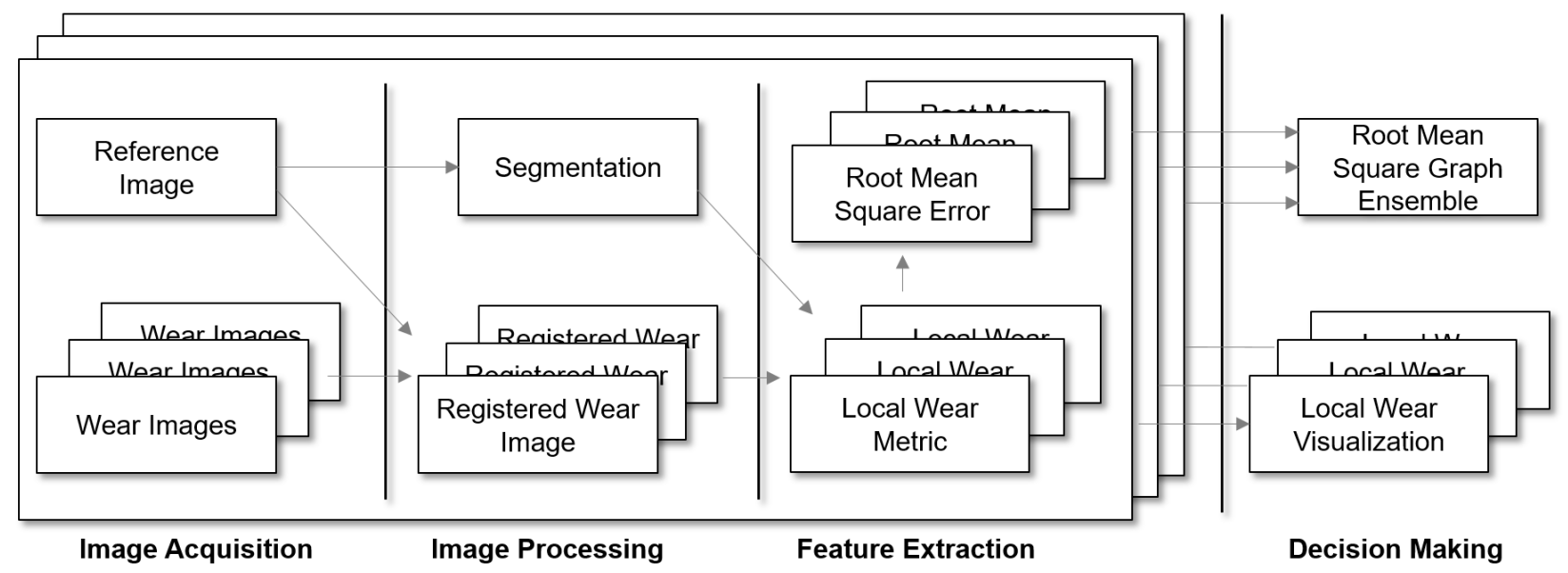

Fig. 1: The industrial vision system pipeline applied to the presented use case: For each cutting tool a data acquisition step is performed to obtain a reference image and images of the cutting tool after different times of use. These images are forwarded in the visual system pipeline to obtain a local wear measurement. For multiple results of these measurement a comparative visualization is presented in this paper.

\section{Image Acquisition}

The Industrial Vision System is applied for determining the flank wear of turning tools (indexable insert). Four indexable inserts with different cutting tool geometries [19, 28] were considered. The wear state of each tool was recorded after successive cutting paths. The material machined was Austempered Ductile Iron (ADI) 900, the cutting tool was made of coated cemented carbide. Further Information on cutting and tool parameters are listed in Table 1.

Before the application of the tool (cutting path of $0 \mathrm{~m}$ ), a reference image of the tool was recorded with a common optical microscope. As can be seen in Fig. 2 left, no wear is detectable at this state. After successive cutting paths of 50m, $150 \mathrm{~m}$ and $250 \mathrm{~m}$ images of the tool were recorded to capture 
Table 1: Specifications of the four considered cutting tools in this study. Example images according to each cutting tool can be found in Fig. 2.

\begin{tabular}{l}
\hline Indexable Insert \\
cemented carbide \\
$\cdot$ grain size: superfine $(0.2-0.9 \mu \mathrm{m})$ \\
$\cdot 89.7 \%$ tungsten carbide $/ 9.6 \%$ cobalt $/$ rest chrome \\
shape: DNMA 150416 \\
corner radius $r_{\epsilon}: 1.6 \mathrm{~mm}$ \\
tool included angle $\epsilon: 55^{\circ}$ \\
wedge angle $\beta$ : 90 \\
cutting edge geometry: \\
- tool 1 : sharp cutting edge \\
- tool 2 : form-factor $\mathrm{K} \approx 1 /$ cutting edge radius $r_{\beta} \approx 25 \mu \mathrm{m}$ \\
- tool 3 : form-factor $\mathrm{K} \approx 1 /$ cutting edge radius $r_{\beta} \approx 55 \mu \mathrm{m}$ \\
- tool 4 : form-factor $\mathrm{K} \approx 0,5 /$ cutting edge radius $r_{\beta} \approx 25 \mu \mathrm{m}$ \\
\hline Coating \\
AlTiN (approx. $2 \mu \mathrm{m})$ \\
\hline Cutting process \\
cooling lubricant: dry \\
cutting speed: $v_{c}=160 \mathrm{~m} / \mathrm{min}$ \\
feed: $\mathrm{f}=0.2 \mathrm{~mm} / \mathrm{U}$ \\
depth of cut: $a_{p}=2 \mathrm{~mm}$ \\
tool cutting edge angle: $\kappa=93^{\circ}$ \\
tool orthogonal rake angle: $\gamma=-6^{\circ}$ \\
tool cutting edge inclination: $\lambda=-6^{\circ}$ \\
\hline
\end{tabular}

the wear state of the tool (Fig. 2). In the figure, also the maximum width of the flank wear land $V B_{\max }$ was detected. As can be seen, this value is subjective and does not give any information on the wear of the whole area of the flank face.

In industrial practice, tools are exchanged when a certain width of the flank wear land is reached. This can result into premature exchange as the flank face might not be worn significantly despite a high width of the flank wear land. On the other hand, it might also be the case that the flank face is severely worn despite of a small width of the flank wear land. Hence an objective, 2-Dimensional wear detection can improve sustainability and economy of cutting processes.

Mathematically, an image $I$ is a two-dimensional matrix of pixels. The matrix has the size of the image resolution, where each pixel can be addressed by its $\mathrm{x}$ and $\mathrm{y}$ coordinate. Each pixel in a colored image holds the three different values encoding the color (red, green and blue). They can be referenced as $(x, y) \cdot r,(x, y) . g$ and $(x, y) . b$. These conventions will be used throughout this paper. Usually, the values of the color components can vary between 0 (no color) and 255 (full color). To ease the calculation, these values are normalized to be located between 0 and 1 .

\section{Image Processing}

After the image acquisition is performed, the resulting set of images can be forwarded to the image processing step of the industrial visual system pipeline. This step is required to correct minor issues in the input images, such as registration and segmentation. Skipping this step would lead to incorrect wear measurement outputs and therefore wrong decision making.

Image processing in general is a widely used term that covers multiple disciplines such as image enhancement, image segmentation and geometric feature extraction [21].

For the available images in the presented case, two tasks belonging to the image processing pipeline need to be solved. First, the cutting tools are not equally aligned in the input images, which requires 


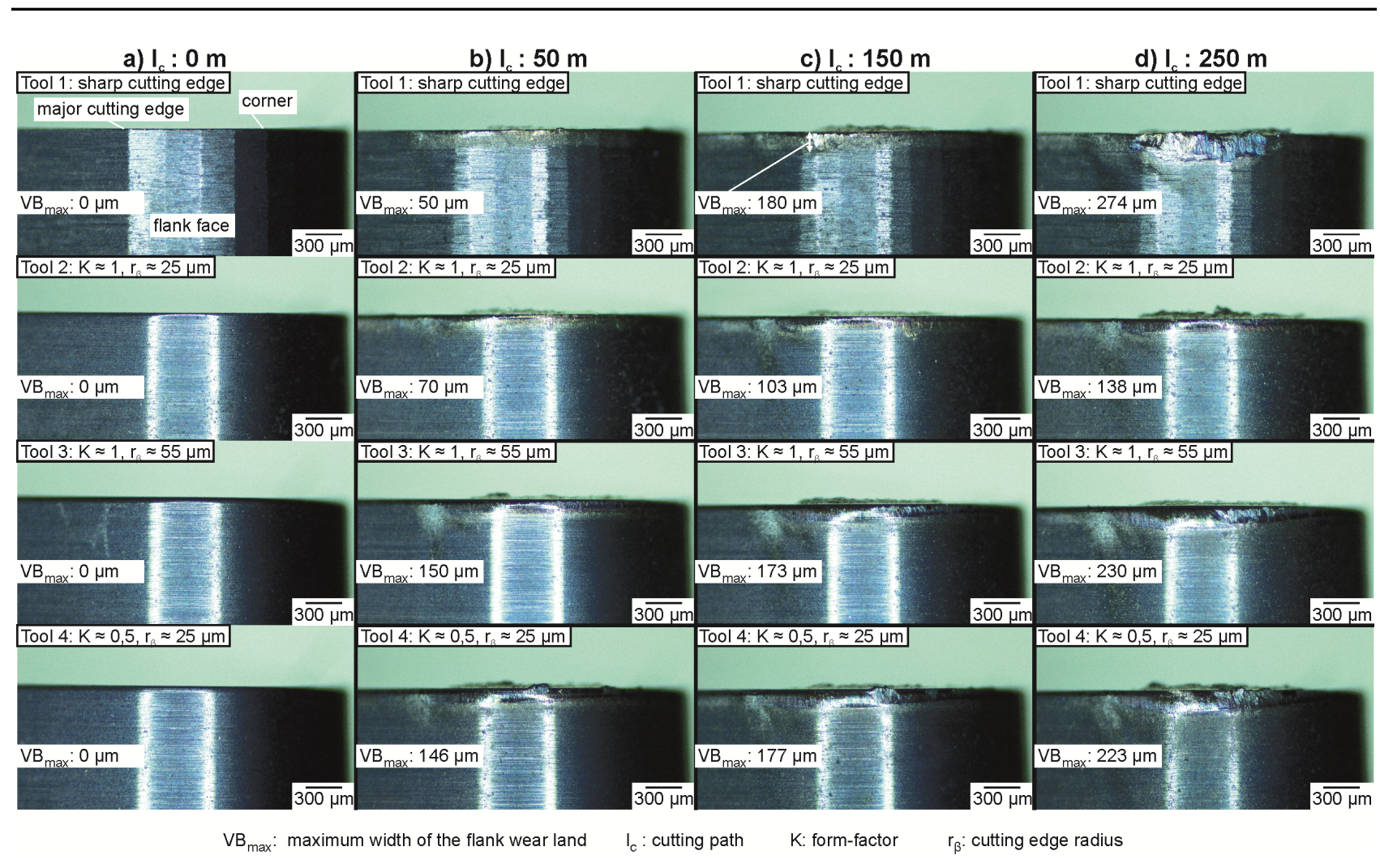

Fig. 2: Images of the four considered cutting tools before their use and after $0 \mathrm{~m}, 50 \mathrm{~m}, 150$ and $250 \mathrm{~m}$.

a correction step called image registration. Second, it is required to identify the region in the image, that belongs to the cutting tool through image segmentation. The following sections will show which algorithms are used in particular and how they perform on the given input image.

Image Registration: As Figure 3 shows, the captured images are not aligned perfectly. This is caused by the manual placement of the cutting tool to obtain an image. For a further comparison of the images they need to be aligned such that these can be compared to the original image of the cutting tool. This problem is known as image registration. There exists a large variety of image registration methods each targeting different settings [22].

In general, all these methods work on two input images: a fixed image and a moving image. The algorithm tries to estimate a transformation of the moving image that maps this moving image to the fixed image as good as possible. Therefore, the algorithm is designed iteratively where an optimization function is used to derive the required transformation. This function works on the basis of a specific metric that determines the overlay of the two images. In this specific case, an enhanced correlation coefficient [23] that is invariant to photometric distortions in contrast and brightness is used.

For each image of a specific cutting tool and its reference image, the image registration process is performed to identify the transformation that matches them as good as possible. A sample registration result can be found in Figure 3. a) displays the original image of the cutting tool before its use. b) Shows the cutting tool used for $150 \mathrm{~m}$. It can be observed, that the tool is shifted to the bottom in this image. c) Shows the $150 \mathrm{~m}$ used cutting tool after the image registration process. The red line indicates that the shift was corrected. Based on this result, the wear of the displayed cutting tool can be estimated by showing the difference between the registered images.

Fuzzy Image Segmentation Classically, a segmentation is defined as the partition of image pixels into different groups, so called segments [24]. This can be accomplished by various different algorithms that work based on different models. All of these algorithms output a second image that tags the original image's pixels according to the segment which they belong to.

Although these algorithms are used in a large variety, they come with one major problem. The fixed assignment of image pixels to classes does not allow communication of the uncertainty of the 


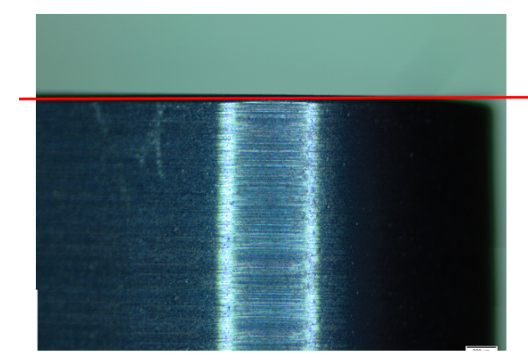

a)

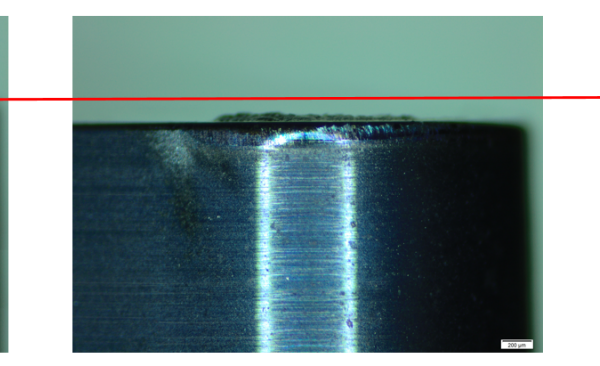

b)

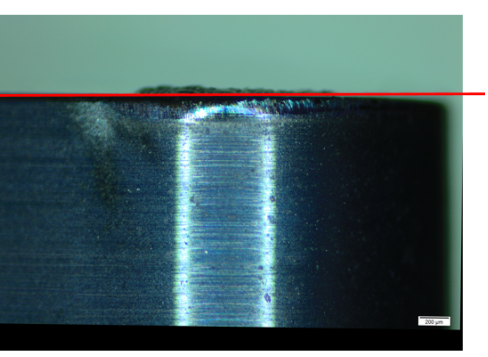

c)

Fig. 3: Example of successful image registration. a) Reference image of cutting tool (fixed image). b) Cutting tool of a) after $150 \mathrm{~m}$ use. The tool is shifted in the image due to the manual placement on front of the camera (moving image). c) Output of image registration process for $a$ ) and b) as input images.

image segmentation process. As real world images are usually affected by various inaccuracies, a fixed segmentation output can contain various classification inaccuracies. In contrast to a fixed segmentation assignment, a fuzzy segmentation assignment is able to communicate the uncertainty of a segmentation process.

The method utilized in this paper is based on an initial user input [25]. With this input, the user contributes his or her knowledge on where the cutting tool and the background are located in the image into the segmentation process. These points are referred to as seed points $s_{\text {edge }}$ and $s_{\text {back }}$. There exist various algorithms which do not require user input. Although they can be run automatically, they often require additional information about the image that needs to be segmented. With the given image samples, this information is not available.

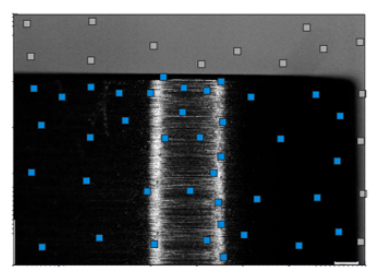

a)

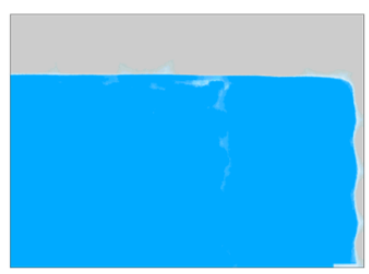

b)

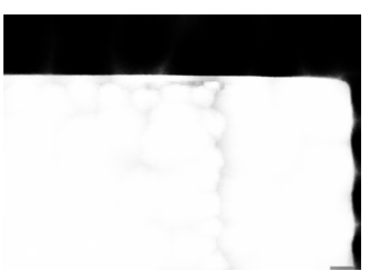

c)

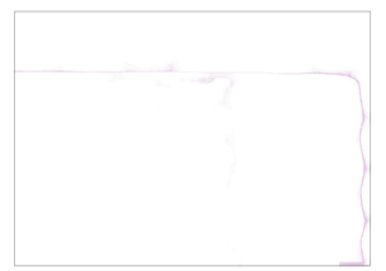

d)

Fig. 4: a) Gray scaled image of cutting edge 3 with the user selected seed points for the cutting edge (blue) and the background (gray). b) Segmentation result color coded with the seed point's colors. c) Weight of cutting edge segment for each pixel (white: full ownership, black: not ownership). This result is forwarded in the visual industrial system pipeline. d) Uncertainty colorcoded with purple highlighting the pixels in the image that cannot be clearly segmented.

Beginning with the user-defined starting points the algorithm assigns each point probability of belonging to the cutting tool or to the background. This is accomplished by measuring the distance from an arbitrary pixel to each starting point. This distance is chosen as the geodetic path in the presented case. In the presented case, we consider all pixels connected to their 4 direct neighboring pixels (top, bottom, left and right). The length of this connection is the difference of the pixel values that are connected. With this setup, the goal is to identify the distance for each pixel to the user defined starting points. Therefore, it is obvious that there are multiple options. From all these options the shortest path is searched. Therefore, the Dijkstra-Algorithm can be utilized [26]. Based on this, for each pixel it is possible to identify the shortest path to one of the starting points for the cutting edge and the background. This distances for an arbitrary pixel $\mathrm{p}$ are referred to as $d_{\text {edge }}(x)\left(d_{\text {edge }}\right)$ and $d_{\text {back }}(x)\left(d_{\text {back }}\right)$. 
Based on these distances, it is possible to assign each pixel a probability whether it belongs to the cutting edge or not using the following equations:

$$
\omega_{\text {edge }}=\frac{\left(d_{\text {edge }}\right)^{-2}}{\left(d_{\text {edge }}+d_{\text {back }}\right)^{-2}}, \omega_{\text {back }}=\frac{\left(d_{\text {back }}\right)^{-2}}{\left(d_{\text {edge }}+d_{\text {back }}\right)^{-2}}
$$

The weights $\omega_{\text {edge }}$ and $\omega_{\text {back }}$ sum up to 1 . The smaller the distance of a pixel to a starting point of the edge the higher the weight gets. On the other hand, this decreases the weight of this pixel for the background. This works for the background class similarly. Based on this calculation, it is possible to obtain a weighted segmentation result for an input image that divides a pixel in two probabilities: first, the probability to belong to the cutting tool and second, the probability of belonging to the background.

Fig 4 shows the segmentation result for the first image of the acquired image series. The seed points are shown in a). The resulting segmentation is colorcoded in b). The weights for the cutting tool are shown in c). These weights are forwarded along the visual industrial system pipeline whereas d) shows the uncertainty of the segmentation result. Purple indicates pixels that cannot be clearly separated into tool or background. In the following calculations of the visual industrial system this is a useful information to enhance the proposed wear measurement.

\section{Feature Extraction - Image Based Wear Measurement}

As shown in Section Acquisition, the image processing step outputs a set of differently long cutting tools registered with their reference image and a segmentation for each reference image determining all pixels that belong to the cutting tool. Based on this input, this paper presents a novel image-based wear measurement. As the wear of a cutting tool is visible in the captured images as a changing upper border of the cutting tool, the goal of the wear measurement is to capture this area specifically. Therefore, a suitable mathematical description of the difference between the reference image to the image of the worn cutting tool is required.

Considering a pixel location $(x, y)$ the difference of an arbitrary pixel color component (in the given case red, green and blue) can be calculated by $D(x, y)_{c}=I_{r e f}(x, y) . c-I_{c u r r}(x, y) . c$, where c can be red, blue or green. Based on this difference, the wear measurement can be defined as:

$$
\text { wear }_{x, y}=(0.21 D(x, y) \cdot r+0.72 D(x, y) \cdot g+0.07 D(x, y) \cdot b) \omega_{\text {edge }}
$$

This outputs a value between 0 (no wear) and 1 (absolute wear) The chosen factors for each color channel are given by the luminosity equation [27]. This equation determines the brightness of a color. The weighting of the different color channels with the luminosity measurement lead to the result that the lightness of the difference is taken into account. As can be seen in the images, the brightness of the cutting tool surface changes if wear occurs. Therefore, luminosity is a suitable way to identify wear in images.

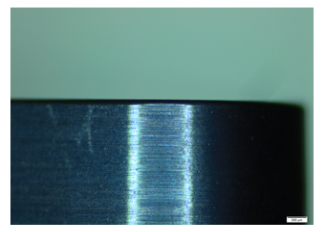

a)

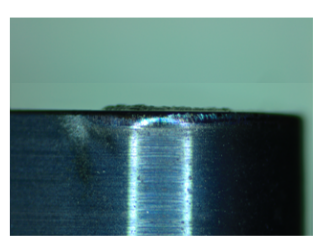

b)

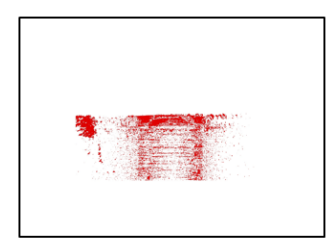

c)

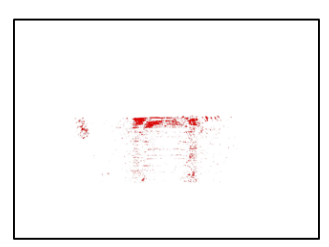

d)

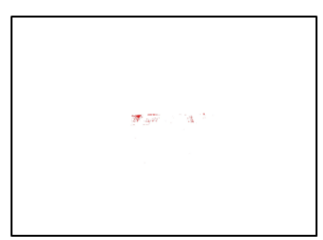

e)

Fig. 5: a) Reference Image of cutting tool 3. b) Cutting tool used $150 \mathrm{~m}$ (registered). Wear measurement of each pixel shown with thresholds c) 0.0 resulting in $\mathrm{RMS}=0.294 \mathrm{~d}) 0.1$ resulting in $\mathrm{RMS}=$ 0.376 and c) 0.2 resulting in $\mathrm{RMS}=0.482$.

The weight of the reference image segmentation works as a stencil that controls the amount of how important the current error is. If the pixel entirely $\left(\omega_{\text {edge }}=1\right)$ belongs to the cutting tool, it needs to be considered completely. If the pixel does not belong to the cutting tool $\left(\omega_{\text {edge }}=0\right)$, this error does 
not need to be considered. This allows the algorithm to neglect image differences in the cutting tool background which do not contribute to the wear of the cutting tool.

As the reference image and the currently considered image can vary in each pixel due to image artifacts, minor image changes are not of interest for the wear estimation. Therefore, a threshold can be used to neglect all wear values that are smaller than the chosen value.

Figure 5 shows the local wears of a cutting tool in use. The darker the red color appears, the higher the error is. The alpha value of the visible red color is depicted based on the resulting wear measure. This results in a transparent red color, when the resulting wear is very low, whereas in contrast to this a high wear measurement out results in an opaque red. The reference image of cutting tool 3 (a)) and the image after $150 \mathrm{~m}$ (b)) are considered in this example. Different thresholds are used to select the visualized wear measurements. c) 0.0, b) 0.1 and e) 0.2 . The images show how minor wear values are filtered. The noise produced by image artifacts is reduced by raising the threshold. The image shows that the area of wear can be clearly identified.

As shown in the example, the presented wear measurement is a local criteria that can be computed for each pixel in an image independently. In contrast to that, a global criteria that determines the wear of the entire tool is required. To achieve this, the root mean square of all wear measurements provides a global measurement of wear for each considered image.

Based on this measurement, it is possible to determine the wear for each image of a cutting tool after a specific time of use in two ways: a quantitative measurement of wear through the root mean square and a visual highlighting of the location of area where the wear occurs.

\section{Decision Making - Visual System}

Tool 4

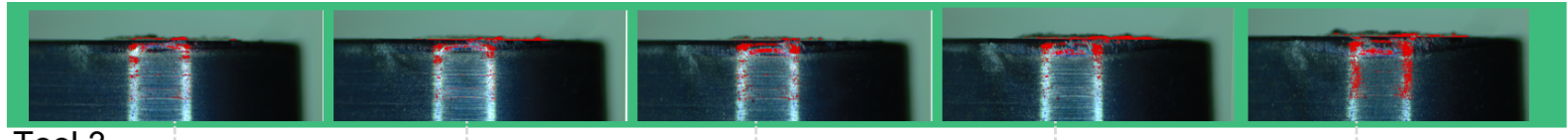

Tool 3

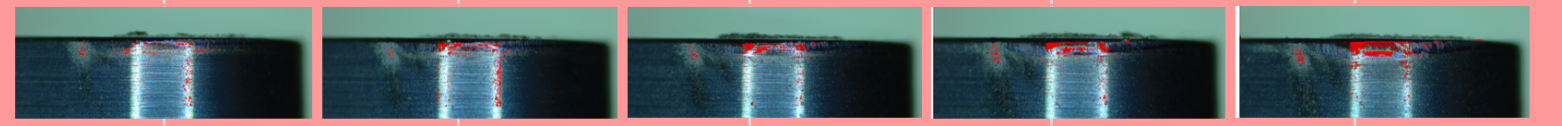

Tool 2

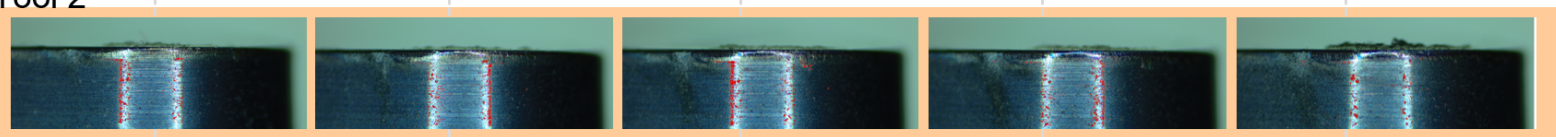

Tool 1
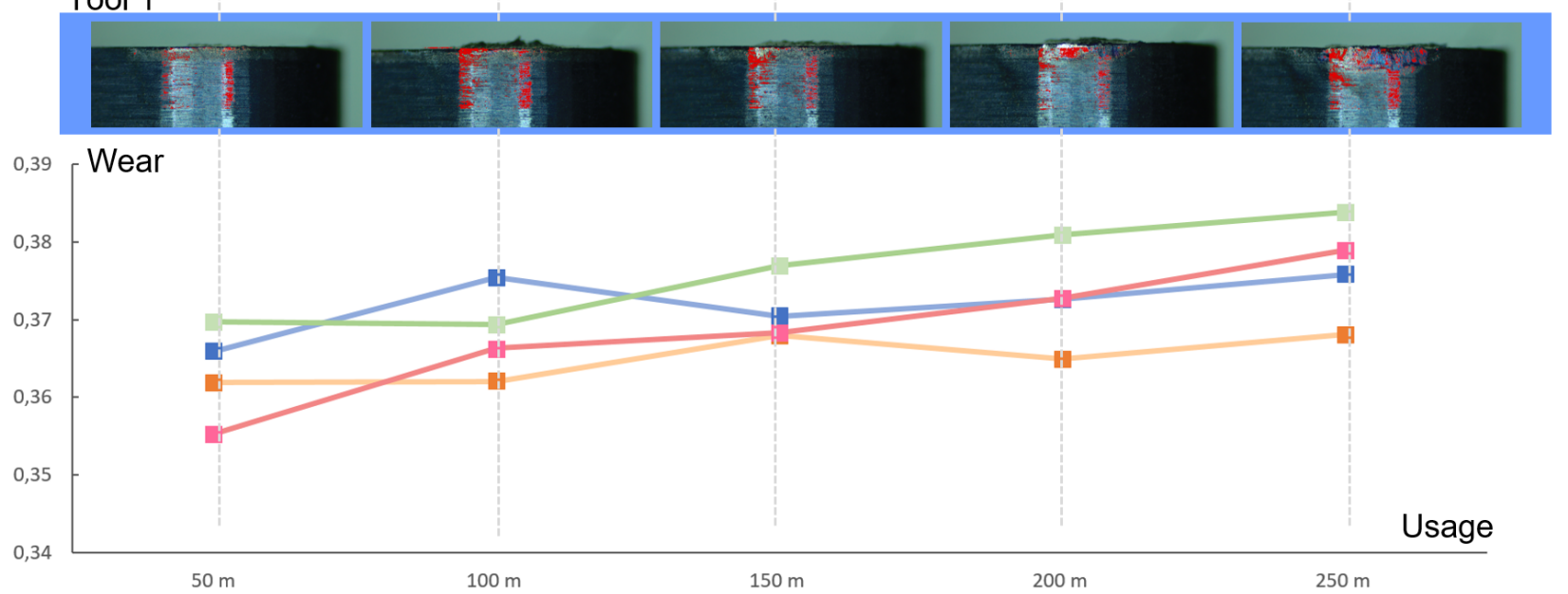

Fig. 6: Resulting visualization for wear behavior of cutting edges 1-4. The visualization consists of two parts. An ensemble root mean square graph showing the rise of wear during the cutting process in an comparative manner. On top of that, the wear measurement for all considered cutting edges is embedded in the original images to highlight the location of wear. 
As shown in Section multiple samples of cutting tool are available that are coated with different shapes. Due the the different shapes, different amounts of wear can be expected [19] . Therefore, a comparative visualization is required to show how the wear of each cutting tool evolves over time and also how the wear evolves depending on the different shape.

Therefore, Fig. 6 shows the resulting interface for the decision maker. The interface is divided into two parts: the ensemble root mean square graph and a stacked view of the wear measurement embedded in the original images.

The root mean square graph plots the root mean square results at each point in time for one cutting tool in one color. Therefore, the resulting plot contains four graphs as four different cutting tool are considered. As can be observed, the rise of wear over time for each cutting tool can be monitored. In addition to that, the root mean square of wear between different types of cutting edges can be seen clearly.

On top of the ensemble root mean square graph, the wear measurements for each pixel are displayed. The visualization solely shows the area where the wear occurs. Instead of solely visualizing the wear measurement output, the measurement is embedded in the registered images of the cutting tool. This results in a highlighting of areas that are affected by wear. This provides an intuitive way of reviewing the wear behavior over time by considering the horizontally aligned images and comparative reviewing of different cutting edge geometries at a specific point in time by considering vertically aligned images. The images have a colored background that has the same color as the corresponding root mean square graph. In addition to that, the position of the images is depending on the time point and its position in the ensemble root mean square graph.

In summary this method implements an entire visual industrial system, that can be used to determine the wear affects of cutting tools at different points in the production process and provide an comparative visualization of effects for different cutting edge geometries.

\section{Discussion}

As Fig 6 shows, the presented visual industrial system is able to generate and visualize the wear of cutting tools in an intuitive and general manner. The system is suitable for all kinds of images independent in size and color. The system solely utilizes the provided image input which makes it independent from further sensors that can be expensive or require maintenance. Still, the additional usage of sensor information is not excluded and could also be incorporated as a color-coding in the visualization.

The utilized image processing algorithm is suitable in the presented case as the results indicate. Still, they increase the computational effort. A real-time usage as desired in on-line scenarios can therefore not be guaranteed and may need further optimizations of the algorithms.

The visualization itself is easy to understand as the available image series are ordered as a twodimensional matrix (x-Axis: time of use, $y$-Axis: tool number). This allows the decision maker to estimate the quality of a coating and compare it to others. The visualization is not restricted to a specific number of time-steps or amount of cutting tools considered. Of course, the complexity of the visualization raises with the number of input images used in the comparison.

The quality of the utilized wear measurement highly depends on the considered images. Anything that is not visible in the image cannot be computed. As this would also not be visible with the naked eye, this precision is sufficient.

In summary, the presented visual system is a powerful tool to calculate and visualize the wear of cutting tools. Further experiments on how accurate the measurement matches sensor-based measurements are subject of further investigation. 


\section{Conclusion}

This paper presented an industrial vision system that is able to use arbitrary images of cutting edges, prepare them with suitable image processing methods and apply a novel wear measurement to identify the amount and location of wear without considering further sensors. The results for multiple wear measurements can be visualized in an intuitive way to compare different cutting edge coatings and review the wear of cutting tools over time. The Vision System can be applied to any cutting tool, i.e. drilling and milling tools can also be evaluated. In future works, the system and the wear measurements will be related to force measurements of the process. The goal is to gain a factor relating the force to the area of flank wear $\left(\mathrm{N} / \mu \mathrm{m}^{2}\right)$ and to define a new abort criterion for tool exchange. This new objective and 2-dimensional abort criterion will help to increase efficiency of cutting processes and to reduce waste.

\section{Acknowledgements}

This research was funded by the German Research Foundation (DFG) within the IRTG 2057 "Physical Modeling for Virtual Manufacturing Systems and Processes".

The authors thank the KSB foundation for their financial support within the research project 1314 "OptiADI - Optimization of tool life during machining of Austempered Ductile Iron (ADI) by specific cutting edge preparation with elastic bonded grinding wheels".

\section{References}

[1] J. Caldeirani Filho and A. E. Diniz: Influence of Cutting Conditions on Tool Life, Tool Wear and Surface Finish in the Face Milling Process Mater. SciElo Brasil Vol.24, p.10-14, No.1, 2002

[2] J. Caldeirani Filho and A. E. Diniz: Methods of Measuring wear-corrosion synergism Elservier Science p.476-484 1995

[3] DOLINŠEK, Slavko, ŠUŠTARŠIČ, Borivoj and KOPAČ, Janez: Wear mechanisms of cutting tools in high-speed cutting processes Elservier Science Vol. 250, Nr. 1, 349-356, 2001

[4] Christina Gillmann, Heike Leitte, Thomas Wischgoll and Hans HagenFrom Theory to Usage:Requirements for successful Visualizations in Applications C4PGV Workshop 2016

[5] K. F. Martin, J. A. Brandon, R. I. Grosvenor, A. Owen A Comparison of in-Process Tool Wear Measurement Methods in Turning Proceedings of the Twenty-Sixth International Machine Tool Design and Research Conference, Springer Vol. 26, p 289-296, 1986

[6] Chen Zhang, Jilin Zhang On-line tool wear measurement for ball-end milling cutter based on machine vision Computers in Industry Vol. 64, Nr.6, p. 708-719, 2013

[7] Luka Cerce, Franci Pusavec, Janez Kopac Novel Spatial Cutting Tool-wear Measurement System Development and its Evaluation Procedia CIRP Vol. 8, Nr. 1, p. 170-175, 2015

[8] S. Ito, S. Sekine, Y. Shimizu, W. Gao, T. Fukuda, A. Kato, and K. Kubota. Measurement of Cutting Edge Width of a Rotary Cutting Tool by Using a Laser Displacement Sensor. IJAT Vol. 8, Nr. 1, p. 28-33, 2014

[9] Arnaud Devillez, S Lesko and W Mozer Cutting tool crater wear measurement with white light interferometry Wear Vol 256., p. 56-65, 2004 
[10] B. Denkena and D. Biermann Cutting edge geometries Precision Engineering Vol. 44, p.236-244, 2014

[11] M.A.F. Ahmada, M.Z. Nuawia, S. Abdullaha, Z. Wahida, Z. Karimb, M. Dirhamsyahc Development of tool wear machining monitoring using novel statistical analysis method, I-kaz Procedia Engineering Vol. 101, 355-362, 2015

[12] Christophe Letot, Roger Serra, Maela Dossevi and Pierre Dehombreux Cutting tools reliability and residual life prediction from degradation indicators in turning process International Journal of Advanced Manufacturing Technology Vol. 86, Nr.1-4, p.495-506 2016

[13] Luka Čerče, Franci Pušavec and Janez Kopač A New Approach to Spatial Tool Wear Analysis and Monitoring Strojniski Vestnik Vol. 61, Nr. 9, p.489-497, 2015

[14] Wenhui Wang, Y.S. Wong and Geok-Soon Hong 3D measurement of crater wear by phase shifting method Wear Vol. 261, Nr.2, p.164-171, 2015

[15] W. Rmili, A. Ouahabi, R. Serra and R. Leroy An automatic system based on vibratory analysis for cutting tool wear monitoring Measurement Vol. 177, p.117-123, 2016

[16] A. Karthik. S. Chandra, B. Ramanoothy and S. Das 3D tool wear measurement and visualisation using stereo imaging International Journal of Machine Tools and Manufacture Vol. 37, Nr. 11, p.1573-1581, 1997

[17] Pan Fua, Weilin Lib, Liang Guo Fuzzy Clustering and Visualization Analysis of Tool Wear Status Recognition Procedia Engineering Vol. 23, p.479-485, 2011

[18] Weilin Li; Pan Fu; Xiaohui Li Visualization Monitoring of Tool Wear States Based on Supervised Dimensionality Reduction and Clustering Procedia Engineering Vol. 8, p.4p1056, 2013

[19] W. Rmili, A. Ouahabi, Roger Serra and René Leroy A survey on industrial vision systems, applications and tools CIRP Annals - Manufacturing Technology Vol. 63, No.1, 201

[20] W. Rmili, A. Ouahabi, Roger Serra and René Leroy A survey on industrial vision systems, applications and tools Measurements Vol. 77, No.1, 2003

[21] Russ, John C. The Image Processing Handbook CRC Press, Inc. 2002

[22] Barbara Zitová and Jan Flusser Image registration methods: a survey IMAGE AND VISION COMPUTING Vol. 21, Nr. 11, p 977-1000, 2003

[23] Evangelidis, Georgios D. and Psarakis, Emmanouil Z. Parametric Image Alignment Using Enhanced Correlation Coefficient Maximization. IEEE Trans. Pattern Anal. Mach. Intell. Vol. 30, $1858-1865,2008$

[24] Nida M. Zaitoun and Musbah J. Aqel Survey on Image Segmentation Techniques Procedia Computer Science Vol. 65, 797 - 806, 2015

[25] Basir, Otman, Zhu, Hongwei and Karray, Fakhri Fuzzy Based Image Segmentation Springer Berlin Heidelberg 101-128, 2003 
[26] Kairanbay Magzhan and Hajar Mat Jani A Review And Evaluations Of Shortest Path Algorithms INTERNATIONAL JOURNAL OF SCIENTIFIC \& TECHNOLOGY RESEARCH Vol.2, Nr.6, p99-104, 2013

[27] Gonzalez, Rafael C. and Woods, Richard E. Digital Image Processing Prentice-Hall, Inc. Vol.3, 2006

[28] J.C. Aurich, C. Effgen, B. Kirsch Cutting edge preparation with elastic bonded superabrasive grinding wheels. CIRP Annals - Manufacturing Technology Vol. 65 Nr. 1, p.329-332, 2016. 